BMJ Open

Ophthalmology

\title{
Incidence, progression and risk factors of age-related macular degeneration in 35-95-year-old individuals from three jointly designed German cohort studies
}

Caroline Brandl (D) ,1,2 Felix Günther, ${ }^{1,3}$ Martina E Zimmermann (D) ,' Kathrin I Hartmann, ${ }^{4}$ Gregor Eberlein, ${ }^{4}$ Teresa Barth, ${ }^{2}$ Thomas W Winkler, ${ }^{1}$ Birgit Linkohr, ${ }^{5}$ Margit Heier, ${ }^{5,6}$ Annette Peters, ${ }^{5,7}$ Jeany Q Li (iD , ${ }^{8}$ Robert P Finger, ${ }^{8}$ Horst Helbig, ${ }^{2}$ Bernhard H F Weber, ${ }^{9}$ Helmut Küchenhoff, ${ }^{3}$ Arthur Mueller, ${ }^{4}$ Klaus J Stark, ${ }^{1}$ Iris M Heid ${ }^{1}$

To cite: Brandl C, Günther F, Zimmermann ME, et al. Incidence, progression and risk factors of age-related macular degeneration in 35-95-year-old individuals from three jointly designed German cohort studies. BMJ Open Ophthalmology 2022;7:e000912. doi:10.1136/ bmjophth-2021-000912

- Additional supplemental material is published online only. To view, please visit the journal online (http://dx.doi. org/10.1136/bmjophth-2021000912).

$\mathrm{CB}$ and $\mathrm{FG}$ contributed equally.

Received 28 September 2021 Accepted 6 December 2021

A Check for updates

(c) Author(s) (or their employer(s)) 2022. Re-use permitted under CC BY-NC. No commercial re-use. See rights and permissions. Published by BMJ.

For numbered affiliations see end of article.

Correspondence to Dr Iris M Heid; Iris.Heid@klinik. uni-regensburg.de

\section{ABSTRACT}

Objective To estimate age-related macular degeneration (AMD) incidence/progression across a wide age range.

Methods and analysis AMD at baseline and follow-up (colour fundus imaging, Three Continent AMD Consortium Severity Scale, 3CACSS, clinical classification, CC) was assessed for 1513 individuals aged 35-95 years at baseline from three jointly designed population-based cohorts in Germany: Kooperative Gesundheitsforschung in der Region Augsburg (KORA-Fit, KORA-FF4) and Altersbezogene Untersuchungen zur Gesundheit der Universität Regensburg (AugUR) with 18-year, 14-year or 3-year follow-up, respectively. Baseline assessment included lifestyle, metabolic and genetic markers. We derived cumulative estimates, rates and risk factor association for: (1) incident early AMD, (2) incident late AMD among no AMD at baseline (definition 1), (3) incident late AMD among no/early AMD at baseline (definition 2), (4) progression from early to late AMD.

Results Incidence/progression increased by age, except progression in 70+-year old. We observed 35-55-yearold with 3CACSS-based early AMD who progressed to late AMD. Predominant risk factor for incident late AMD definition 2 was early AMD followed by genetics and smoking. When separating incident late AMD definition 1 from progression (instead of combined as incident late AMD definition 2), estimates help judge an individual's risk based on age and (3CACSS) early AMD status: for example, for a 65-year old, 3-year late AMD risk with no or early AMD is $0.5 \%$ or $7 \%$, 3-year early AMD risk is $3 \%$; for an 85 -year old, these numbers are $0.5 \%, 21 \%, 12 \%$, respectively. For CC-based 'early/intermediate' AMD, incidence was higher, but progression was lower.

Conclusion We provide a practical guide for AMD risk for ophthalmology practice and healthcare management and document a late AMD risk for individuals aged $<55$ years.

\section{INTRODUCTION}

Age-related macular degeneration (AMD) is a degenerative disorder affecting the complex of photoreceptors, retinal pigment epithelium

\section{Key messages}

What is already known about this subject?

While there are numerous epidemiological studies with cross-sectional data on age-related macular degeneration (AMD), population-based longitudinal studies and estimates for incidence and progression for AMD are scarce.

\section{What are the new findings?}

- We report incidence of early and late AMD as well as progression and respective risk factors covering a particularly wide age spectrum in three jointly designed cohort studies. By integrating our results with estimates from four other European cohorts, we provide a comprehensive view on incident late AMD. In our data, also younger individuals aged 35-55years with baseline early AMD exhibited at least a sevenfold increased 18-year cumulative risk for late AMD compared with individuals at the same age without AMD.

\section{How might these results change the focus of} research or clinical practice?

Our practical guide for ophthalmologists helps judge an individual's risk for late AMD based on age and early AMD status, which is also relevant for healthcare management and clinical trial design. Our results might draw more attention to AMD in the young.

(RPE) and choroid in the central retina. ${ }^{1}$ It represents the leading cause of central vision loss in the elderly populations of industrialised countries. ${ }^{1}$ Due to population ageing, the number of affected individuals has been

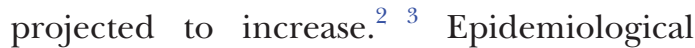
measures such as prevalence, incidence and progression are key for healthcare management and individuals' risk assessment.

While there are numerous populationbased studies with one-time assessment of AMD (cross-sectional studies) estimating 
disease prevalence, ${ }^{24-7}$ there are only few studies with multiple AMD assessments longitudinally. ${ }^{28} \mathrm{~A}$ recent meta-analysis of European cohort studies had included 22 studies on AMD prevalence, but only four studies on AMD incidence. ${ }^{2}$ In Germany, population-based AMD incidence data are limited to the Gutenberg Health Study with 5-year follow-up. ${ }^{9}$

The current gold standard in epidemiological studies to document early/intermediate and late AMD stages is the grading of colour fundus images of the central retina. On colour fundus images, early AMD is characterised by differently sized yellowish accumulations of extracellular material (drusen) between Bruch's membrane and the RPE or between the RPE and the neurosensory retina. Other features of early/intermediate AMD are RPE abnormalities, including depigmentation or increased amount of pigment. ${ }^{1}$ Late AMD can manifest as an atrophic form known as geographic atrophy (GA) or a macular neovascular (MNV) complication characterised by choroidal/(sub)retinal ingrowth of diseased blood vessels and bleeding. ${ }^{10}$

While the definition of late AMD is fairly similar across clinical and epidemiological studies, classification systems for early AMD differ in terms of graded features and categories. ${ }^{11}$ When comparing the Three Continent AMD Consortium Severity Scale (3CACSS) ${ }^{12}$ and the Clinical Classification (CC) ${ }^{13}$ substantial differences in early AMD prevalence were documented ( $17 \%$ vs $44 \%$, respectively). ${ }^{7}$ A recent assessment of the performance of several classification systems showed that each system had its merits in different settings ${ }^{11}$ and that 3CACSS performed best in predicting progression to late AMD. ${ }^{11} 12$

Further challenges pertain to study design: (1) as AMD is frequent only in the elderly, studies in younger adults require long-term follow-up to observe a sufficient number of events, particularly for late AMD and (2) studies in the old-aged require a study programme meeting the needs of the elderly and a shorter follow-up period to avoid high loss from mortality. This renders it difficult to provide AMD cohort data across a wide age range and epidemiologic measures on AMD in a systematic fashion.

We aimed to provide estimates for early and late AMD incidence as well as for progression from early to late AMD in a wide age spectrum. Making use of the research platforms of KORA (Kooperative Gesundheitsforschung in der Region Augsburg) and AugUR

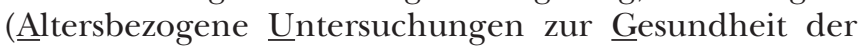
Universität Regensburg), we have set up AMD investigation in three population-based cohort studies in parallel. The joint design enabled harmonisation of colour fundus imaging, AMD assessment, AMD grading (3CACSS, CC) and the optimisation of follow-up length according to participants' age: 18-year follow-up for younger adults (KORA-Fit, 35-55 years), 14-year follow-up for older adults (KORA-FF4, 55-75 years) and 3-year follow-up for the old aged (AugUR, 70-95 years). The KORA and AugUR studies are also highly comparable in terms of recruiting in middle-sized cities in Bavaria, Southern Germany, with participants having similar lifestyle, environment and genetic background. ${ }^{6}{ }^{14}$ This enabled us to observe 1513 individuals aged 35-95 years at baseline and $\sim 6000$ person-years, to estimate early/late AMD incidence and progression, and to quantify relative risk from well-established AMD risk factors like age, sex, smoking, high-density lipoprotein cholesterol (HDL-C) and genetic profile. ${ }^{1516}$

\section{SUBJECTS AND METHODS}

Study populations and study samples

KORA is a research platform recruiting from the population aged 25-74 years living in/around Augsburg, Germany, a city with $\sim 300000$ inhabitants. ${ }^{17}$ Details have been described previously. ${ }^{17}{ }^{18}$ In brief, the present analysis is based on the fourth KORA survey (KORAS4, conducted 1999-2001, $\mathrm{n}=4261$, response $=67 \%) .{ }^{19}$ KORA-S4 was conducted in several study centres, one of them equipped for colour fundus imaging, rendering 2842 of the 4261 KORA-S4 participants eligible for the fundus sub-study, of which 2840 participated as reported previously. ${ }^{6}$

The follow-up in the KORA-S4 fundus substudy contained two parts. First, a 14-year follow-up (KORAFF4, 2013/14) $)^{20}$ included 1653 participants aged 55-74 years with baseline fundus imaging. Of these, 678 individuals obtained follow-up fundus images (follow-up net response $60 \%$; online supplemental table 1A). Second, an 18-year follow-up (KORA-Fit, 2018/19) included 1831 participants aged 35-55 years with baseline fundus imaging. Of these, 856 obtained follow-up fundus images (follow-up net response $61 \%$; online supplemental table 1B).

AugUR is a research platform recruiting from the mobile elderly population in/around Regensburg, Germany, a study region with $\sim 330000$ inhabitants of mostly Caucasian ancestry comparable to KORA in terms of size, living and environmental conditions as well as genetic background. Study recruitment and conduct were designed according to the procedures from KORA as reported previously. ${ }^{14}$ We here report on the first of two AugUR baseline surveys (AugUR1-BL, 2013-2015 n=1133) as described previously. ${ }^{7}$ A 3-year follow-up (applied the same procedures (AugUR1-FU1, 2016-2018); all baseline participants were eligible and reinvited. AugUR1-FU1 included 788 participants with fundus imaging (netfollow-up response 77\%; online supplemental table 1C). Although follow-up response was high, response at baseline indicated selection towards the mobile elderly as described previously. ${ }^{7}$

The recruitment procedures were the same for KORA $^{21}$ and AugUR, ${ }^{14}$ since AugUR was designed after the example of KORA: local registries were asked to draw a random sample of individuals residing in the study area (Augsburg or Regensburg, respectively, and selected counties) and having a birth year that met study requirements (25-74 years for KORA, 70+years for AugUR); 
these individuals were asked to participate by a mailed written invitation letter. For KORA, a German passport was required. There were no a priori exclusion criteria.

\section{Patient and public involvement statement}

It was not appropriate or possible to involve patients or the public in the design, or conduct, or reporting, or dissemination plans of our research.

\section{Assessment of participant characteristics and risk factors at baseline}

Data assessment of KORA and AugUR was highly comparable, since KORA served as model when designing the AugUR study. Details on baseline data collection have been described elsewhere. ${ }^{67141822}$ In brief, information on lifestyle factors and metabolic parameters was gathered via a standardised face-to-face interview, medical examinations by trained medical staff and laboratory measurements from blood (online supplemental text 1). KORA and AugUR study participants were genotyped and genetic information was imputed. Based on 50 of 52 variants reported for late AMD, ${ }^{15}$ we computed a genetic risk score (GRS) by adding the dosages of AMD risk alleles, weighed by the respective variant's published effect size ${ }^{15}$ (online supplemental text 1). Askimed (http://www.askimed.com/) and SAS were used for data management.

\section{AMD classification based on colour fundus images}

Colour fundus photography of the central retina and AMD feature assessment were conducted as described previously $^{67}$ (online supplemental text 2 ). For each eye with gradable colour fundus imaging, AMD status was classified by 3 CACSS $^{12}$ and CC. ${ }^{13}$

All images, from baseline and follow-up in the three cohorts (KORA-S4, KORA-FF4, KORA-Fit, AugUR1-BL, AugUR1-FU1), were graded for AMD features by the same experienced and trained ophthalmological consultants (CB and TB) as described before ${ }^{67}$ Further details on double grading, inter-rater reliability and baseline versus follow-up comparisons are provided in online supplemental text 3 .

Assessed features included presence of drusen and pigment abnormalities (hyperpigmentation or depigmentation), drusen size category (small, intermediate, large), total drusen area and presence of GA and/or MNV. Then, we transferred this information into the AMD status according to the 3CACSS and CC classification systems as 'no' (absence of 'early' or 'late'), 'early' (3CACSS: mild, moderate or severe early; CC: early, intermediate) or 'late' AMD (details described in online supplemental text 2) and previously. ${ }^{67}$

The AMD status of a person was derived as the AMD status of the eye with the more severe AMD stage ('worse eye') when both eyes were gradable, or as the status of the one available eye otherwise. We analysed individuals with at least one eye gradable at baseline and at follow-up.

\section{Defining four types of events}

All participants with AMD status assessed at baseline and follow-up were included in the present analysis. For each participant, we defined four types of 'events' based on the observed transition from baseline to follow-up AMD status: (1) 'incident early AMD' when the person had 'no AMD' at baseline and 'early AMD' at follow-up, (2) 'incident late AMD (definition-1)' for 'no AMD' at baseline and 'late AMD' at follow-up, (3) 'incident late AMD (definition 2) ' for 'no AMD' or 'early AMD' at baseline and 'late AMD' at follow-up and (4) 'progression from early to late AMD' for 'early AMD' at baseline and 'late AMD' at follow-up. Analyses were restricted to the respective 'persons at risk': (1) individuals without AMD at baseline for incident early AMD or incident late AMD (definition 1), (2) individuals with no or early AMD at baseline for incident late AMD (definition 2), (3) individuals with baseline early AMD for progression.

\section{Statistical analyses}

Statistical analyses were carried out in R, V.3.6.3 (R Core Team, 2020) and IBM SPSS Statistics, V.25 (IBM). Descriptive statistics were derived for participants in this longitudinal analysis and those lost-to-follow-up, using logistic regression to test for differences.

We computed cumulative incidence and progression estimates, per study and also by refined age-groups within study, as the number of 'events' divided by the number of 'persons at risk' (online supplemental text 4). Since the classification of 'early AMD' differs between 3CACSS and $\mathrm{CC}$ and, consequently, also the classification of 'no AMD' (ie, absence of early and late AMD), we computed cumulative incidence and progression for 3CACSS-based AMD and CC-based AMD. We compared the two classifications of 'early AMD' by positive predictive value (PPV) for late AMD and area under the receiver operating curve (AUROC; online supplemental text 4). We estimated the association of established risk factors; age, sex, smoking, HDL-C and GRS with incident early AMD, incident late AMD (definition 2) or progression, per study, via multiple logistic regression. ORs were tested for difference from unity (online supplemental text 4 ).

For comparison between our studies and to published studies, we estimated incidence and progression rates per 1000 person-years by study and refined age-groups (online supplemental text 4). The number of events per age-group derived from the individual's baseline age assuming event onset in the middle of follow-up. We conducted sensitivity analyses assuming that events occurred at $2 / 3$ of follow-up. We used incidence/ progression rates to derive 1-year and 3-year cumulative incidence/progression according to $1-\exp (-t *$ rate $)$, where rate is the rate of the respective event and $t$ is the number of years across which events were accumulated (here: 1 or 3 years). We did this by age-group and study and combined across the three studies for the overlapping age-groups. 


\section{RESULTS}

\section{Participant characteristics}

Gradable images for AMD for at least one eye at baseline and follow-up were available for 1513 individuals (2901 eyes at baseline, 2869 at follow-up), which comprised the analysed sample (KORA-Fit $\mathrm{n}=506$, KORA-FF4 $\mathrm{n}=350$, AugUR $\mathrm{n}=657$ ). Analysed participants were $50 \%$ women and baseline age ranged from 35 to 95 years (online supplemental table 2). Net follow-up response was $61 \%, 60 \%$ and $71 \%$, respectively (online supplemental table 1). Individuals not participating in follow-up were slightly older and with a slightly less healthy lifestyle (more current smokers, less physically active, higher BMI), but otherwise comparable to the analysed sample, particularly in terms of baseline AMD status (online supplemental table 2). The three jointly designed AMD studies covered the younger, the older and the old-aged adult population (baseline age 34-55 years, 54-75 years, 70-95 years for KORA-Fit, KORA-FF4, AugUR), followed for 18, 14 and 3 years, respectively (age at follow-up 53-73 years, 69-88 years, 73-98 years, online supplemental figure 1 ).

\section{Cumulative incidence and progression differ between classification systems}

We estimated cumulative AMD incidence and progression by study and refined age-group for the two classification systems, 3CACSS-based and CC-based early AMD ('mild/moderate/severe early' AMD or 'early/intermediate' AMD, respectively). We found lower cumulative incidence for 3CACSS-based early AMD compared with CC-based, but higher progression (eg, KORA FF4: 14-year early AMD incidence $13.9 \%$ vs $41.5 \%$, progression $43.8 \%$ vs $18.5 \%$, respectively; table 1, online supplemental table 3). Late AMD incidence was similar for definition 1, despite the different group at risk (ie, 'no AMD' as absence of early/late AMD), and exactly the same for definition 2 (as expected, group at risk defined as no/early AMD).

The higher progression from 3CACSS-based early to late AMD compared with CC-based early AMD was also reflected by higher PPVs in the three-category view (online supplemental figure 2A). When extending this to the five-category view for refined AMD categories (3CACSS: no, mild early, moderate early, severe early, late AMD; CC: no AMD, age-related changes, early, intermediate, late AMD), we found a consistent increase in PPVs by increased 3CACSS categories in all three studies, but a PPV near zero for lower CC categories rendering these rather uninformative (online supplemental figure $2 \mathrm{~A})$. When evaluating the ability of the two system's no/ early AMD categories to discriminate for future late AMD development via AUROC, we found good performance for both systems (AUROC 0.72 to 0.87 , online supplemental figure 2B). 3CACSS showed an advantage for old-aged in the three-category view. However, CC-based early AMD identified some younger/older individuals who progressed to late $\mathrm{AMD}$, which was overlooked by
3CACSS (one of eight late AMD cases in younger, 3 of 14 in older individuals); this was at the cost of additional 'false-positives' (reduced specificity, online supplemental figure 2B). This pattern was also seen in the five-category view. We focused the following on 3CACSS.

Comparing risk factors association for incident early, incident late AMD and progression

We evaluated association of risk factors assessed at baseline (age, sex, smoking, ex-smoking, HDL-C, GRS) with 3CACSS-based cumulative incidence of early and late AMD (definition 2) by study via logistic regression. We also evaluated association with progression in the old aged (AugUR; not in KORA-Fit, KORA FF4 due to sparse numbers). We found the following (figure 1, online supplemental table 4): (1) GRS and age were consistently associated with increased odds for incident early and incident late AMD, (2) for progression (in old aged), GRS showed significant association, but not age, (2) smoking was associated with increased odds for incident late AMD and, for old-aged, also for progression, but not for incident early AMD, (3) baseline early AMD conveyed the most consistent and highest risk for late AMD across studies without and with GRS as covariate (OR from 21.0 to $57.7 ; \mathrm{p}<0.002)$.

The ORs of the GRS were fairly stable across models and studies, higher for incident late AMD (OR 1.9-4.5 per $5 \%$ of maximum possible GRS) than for early AMD $(\mathrm{OR}=1.5-1.9)$, and $\mathrm{OR}=2.5$ for progression in old aged. For current smoking, we found not only remarkably high ORs (for incident late AMD and, in old-aged, for progression) but also high uncertainty. We found no association for sex, former smoking or baseline HDL-C. The lack of HDL-C association with AMD was not due to adjusting for the GRS, which includes HDL-C-associated genetic variants (in/near LIPC, CETP genes), since the lack was also observed without GRS as covariate.

\section{Rates and 3-year risk of incidence and progression as a practical guide for practitioners}

To compare estimates between studies with different follow-up lengths, we computed rates per 1000 personyears by study and refined age-group, focused on 3CACSS (15830 person-years overall). This revealed a clear increase by age in the rates of incident early AMD and incident late AMD definition 2 (figure 2A,C, online supplemental table 5A,C). There was almost no increase in rates of incident late AMD definition 1 above the age of 60 years (figure 2B, online supplemental table 5B). Rates of progression also increased by age in the younger and older, but not in old aged (figure 2D, online supplemental table 5D). For these analyses, we assumed the observed event to have occurred at half of follow-up time, but sensitivity analyses assuming occurrence at $2 / 3$ provided very similar results (online supplemental table $6)$.

Incidence and progression rates are not fully intuitive for interpretation, but can be transformed to, for 
Table 1 Cumulative estimates for incident early AMD, incident late AMD, and progression by study and refined age groups

(A) Cumulative incidence of early AMD

(B) Cumulative incidence of late AMD (definition 1)*

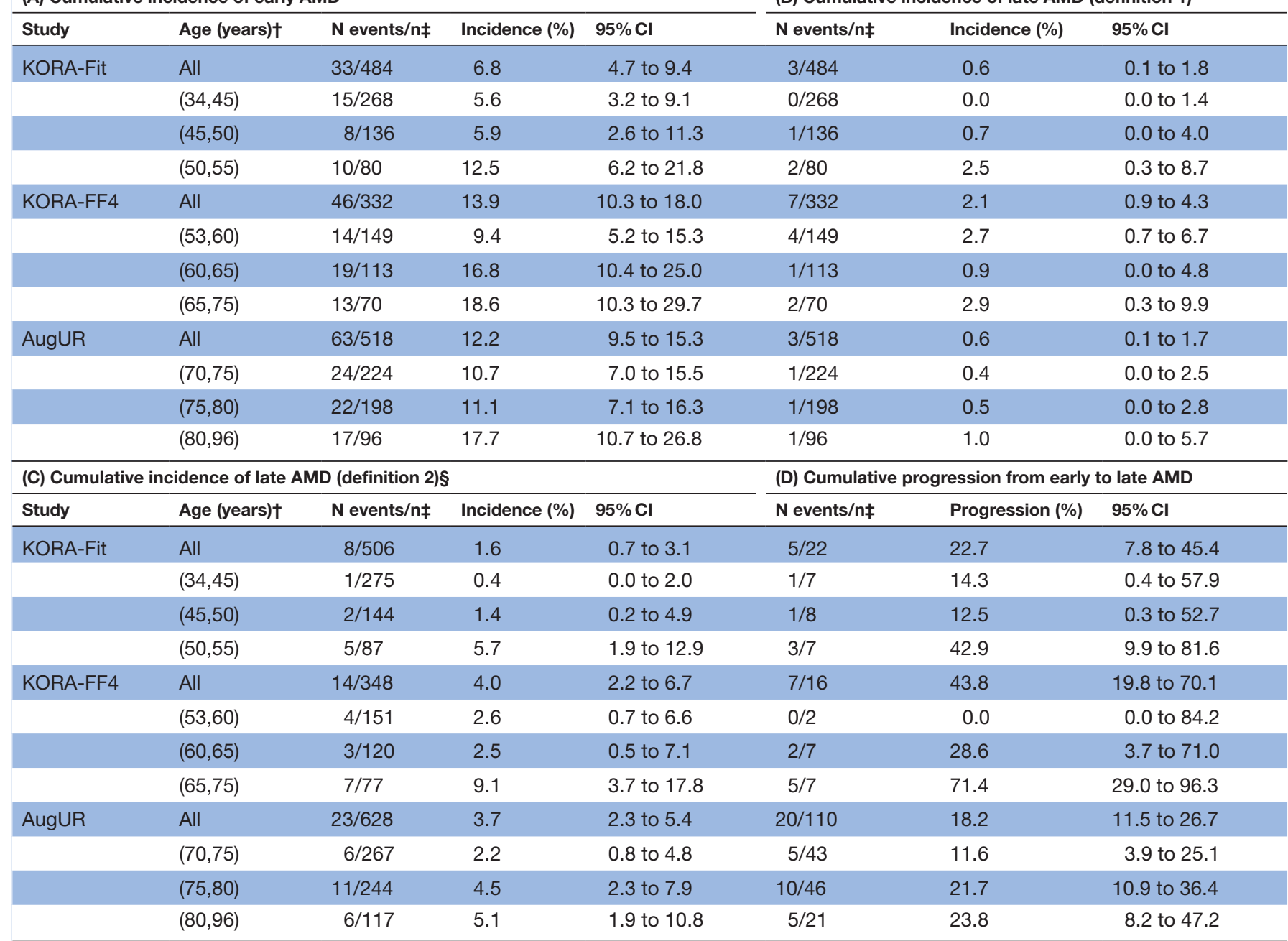

Shown are 18 year, 14 year, and 3 year cumulative estimates and $95 \% \mathrm{Cl}$ for incidence and progression for the three studies (KORA-Fit, KORA FF4, AugUR, respectively), overall and by refined age groups. For AMD classification, 3CACSS12 was applied here (analogous estimates for CC36 in online supplemental table 3 ).

${ }^{*}$ Considering individuals with no AMD at baseline (definition 1).

$\dagger$ Age at baseline.

$\ddagger$ Number of events/number of persons at risk.

§Considering individuals with no or early AMD at baseline (definition 2).

AMD, age-related macular degeneration; AugUR, Altersbezogene Untersuchungen zur Gesundheit der Universität Regensburg; 3CACSS, Three

Continent AMD Consortium Severity Scale; KORA-Fit / KORA-FF4, Kooperative Gesundheitsforschung in der Region Augsburg.

example, 3-year cumulative incidence and progression (Methods). The joint analysis across the three studies allowed us to cover age-groups from 35 to 100 years. We derived a side-by-side comparison of 3-year risk for each of the four events (figure 3A-D), with three of these particularly informative given a person's age and early AMD status: risk of early or late AMD (definition 1) among individuals without AMD (figure 3A,B) and risk of late AMD among individuals with early AMD (ie, progression, figure 3D). For example, our data suggested that an individual aged 65 years without AMD had a 3\% risk to develop early AMD within the next 3 years and a $0.5 \%$ risk to develop late AMD; when the 65-year-old had early AMD, their 3-year risk to progress to late AMD was $7 \%$. For an individual aged 85 years, the numbers were $7 \%, 0.5 \%$ and $21 \%$, respectively. Interestingly, our data indicated that the 65-year-old and the 85-year-old without early AMD have comparable late AMD risk (ie, $0.5 \%$ ). While these estimates are subjected to uncertainty, this can be a practical guide to help judge an individual's risk by age and early AMD status.

Features of early AMD in younger adults are predictive for late AMD development

AMD is commonly defined as a disease-affecting individuals in their sixth decennium and older. In the cross-sectional evaluation of KORA-S4, we had previously detected features resembling early AMD in participants who were markedly younger (as young as $<30$ years). ${ }^{6}$ This had raised the question, whether the detected features were truly AMD-related or artefacts mimicking AMD. For these younger adults with early AMD features, we were 
A

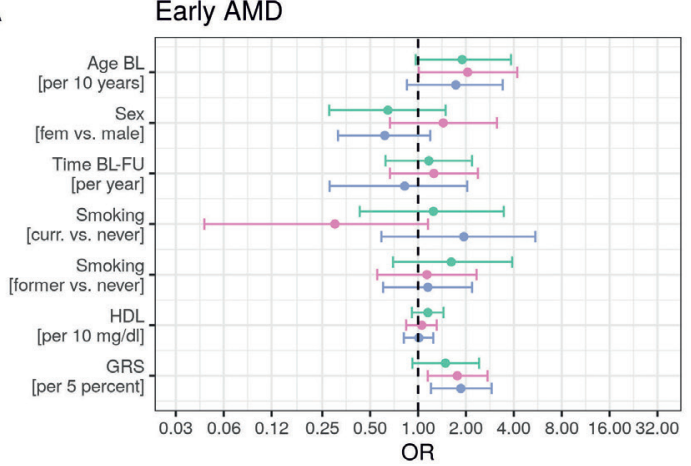

B

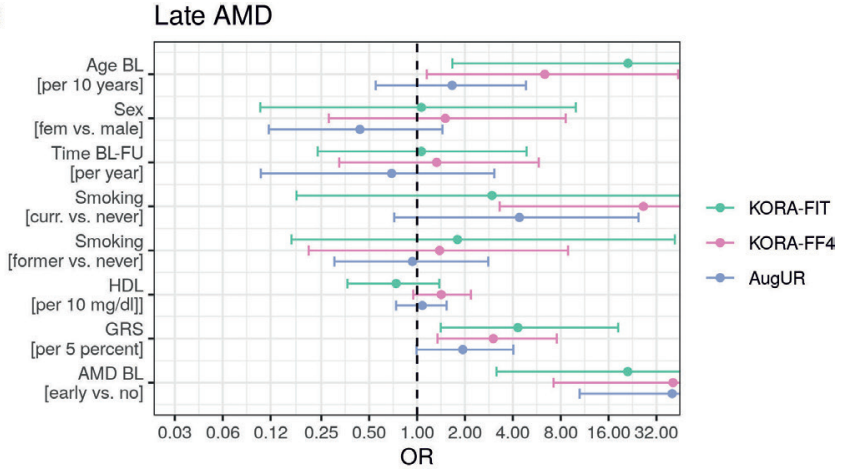

C

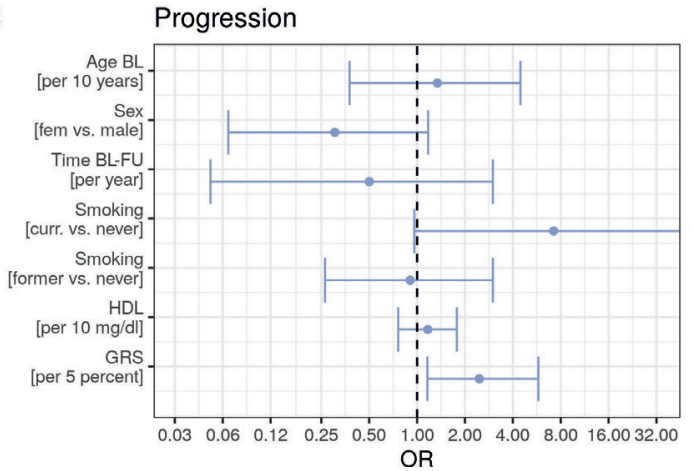

Figure 1 Risk factor association with incident early agerelated macular degeneration (AMD), incident late AMD and progression by study. We conducted logistic regression including age, sex, follow-up time, smoking, former smoking, high-density lipoprotein cholesterol (HDL-C), genetic risk score (GRS) and early AMD at baseline (BL) (as applicable). Shown are OR and $95 \%$ Cls for (A) incident early AMD, (B) incident late AMD among those with no or early AMD at baseline (definition 2) and (C) progression for AugUR. For AMD classification, 3CACSS was applied. Detailed results also from other models are shown in online supplemental table 4). AugUR, Altersbezogene Untersuchungen zur Gesundheit der Universität Regensburg; KORA-Fit / KORAFF4, Kooperative Gesundheitsforschung in der Region Augsburg; FU, follow-up.

now able to assess their risk to progress to late AMD using the 18-year follow-up of KORA-Fit (35-55 years of age at baseline, 53-73 years at follow-up, median follow-up time 18 years): $22.7 \%$ of these progressed to late AMD ( $n=5$ of 22; online supplemental table 1D), compared with $0.6 \%$ among those without baseline AMD ( $\mathrm{n}=3$ of 484 ). This yielded an unadjusted OR of 47.2 for the 18-year cumulative progression to late AMD comparing individuals with baseline early AMD with those AMD-free and an OR of 35.0 (95\% CI 7.4 to 166.0 , p value $<0.0001$ ) when adjusting for age and follow-up time. When regrading the AMD status of these 22 individuals, we confirmed baseline early AMD of all 22, late AMD at follow-up for the 5, and early AMD for the other 17 .

Our derived 3-year risk of disease and progression demonstrates that a 45-year-old without early AMD has a $0.5 \%$ risk to develop early AMD within 3 years (figure $3 \mathrm{~A}$ ) and a practically 0 risk for late AMD (figure 3B); a 45-year-old with early AMD has a $2 \%$ risk of progressing to late AMD within 3 years (figure 3D). Such a person might be underdiagnosed, if there is no attention for these early AMD features also in younger individuals and limited awareness that they can progress to late AMD.

\section{DISCUSSION}

We conducted three population-based, jointly designed cohort studies with $>1500$ individuals aged 35-95 years at baseline and an 18-year, 14-year or 3-year follow-up, respectively, and derived cumulative estimates for AMD incidence and progression. For 3CACSS compared with CC classification, we found lower incidence of early AMD, but higher progression to late AMD, prompting us to focus on 3CACSS for most of the analyses. Incident and progression rates were estimated by age-groups and the corresponding 3-year risk estimates provide a practical guide for an individual's risk given age and early AMD status. Importantly, we found $22.7 \%$ of the 35-55year old with early AMD at baseline to have progressed to late AMD within 18 years of follow-up. Our systematic analysis of AMD risk factors confirmed age, genetics and current smoking and allowed for a direct comparison of those AMD risk factor associations with incident early and late AMD, and also-focused on the high-aged-for progression.

Our data are unique in its wide age range, covering 35-95 years at baseline and 35-100 years for rate estimation, with a longer follow-up of the younger and older to capture a sufficient number of events as well as a shorter follow-up in the old aged to avoid high loss by mortality.

A clear strength of our data is the highly standardised study procedures across the three studies. Data assessment of KORA and AugUR was highly comparable, since KORA served as model when designing the AugUR study. AMD grading of all colour fundus images-across studies as well as across baseline and follow-up-was performed by the same trained ophthalmologists. Moreover, mild pharmacological mydriasis was applied in the majority of participants aged $>70$ years, to ascertain high image quality despite old-age-related smaller pupil size. ${ }^{7}$

Our results are also unique by our side-by-side view of estimates for four types of events: incident early AMD next to two definitions of incident late AMD and progression. Most published estimates on incident late AMD were computed among individuals with no 

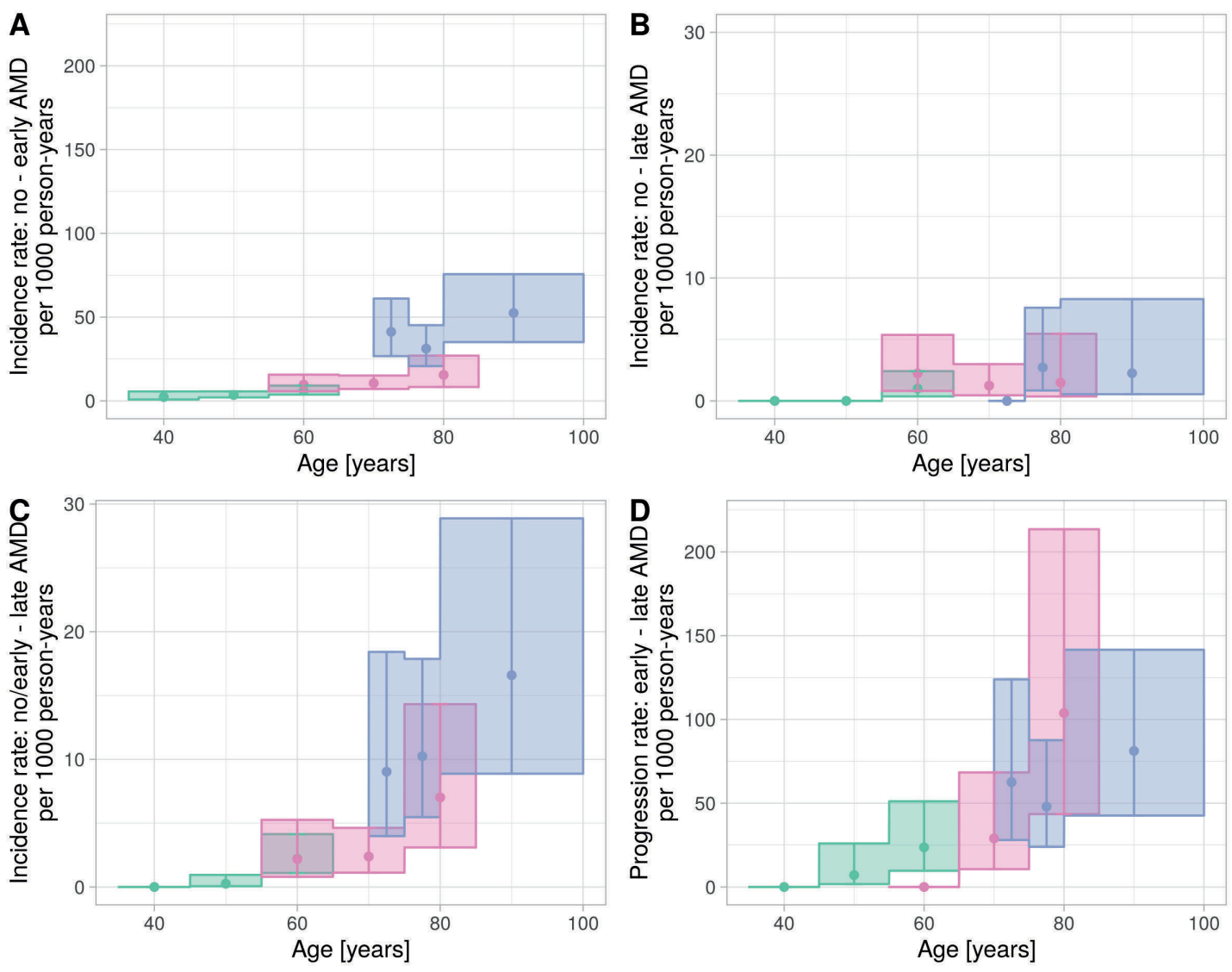

KORA-FIT

KORA-FF4 AugUR

Figure 2 Rates per 1000 person-years for incident early AMD, incident late AMD and progression by study and refined age groups. Rates per 1000 person-years were derived by 10-year age groups (KORA-Fit, KORA FF4) or 5-year age groups (AugUR) and assuming that the event occurred in the middle of the follow-up time. Shown are estimated rates and $95 \%$ Cls for (A) incident early AMD, (B) incident late AMD among individuals with no AMD at baseline (definition 1), (C) incident late AMD among individuals with no or early AMD at baseline (definition 2) and (D) progression from early to late AMD. For AMD classification, 3CACSS was applied. Note the different y-axes scales. AMD, age-related macular degeneration; AugUR, Altersbezogene Untersuchungen zur Gesundheit der Universität Regensburg; KORA-Fit / KORA-FF4, Kooperative Gesundheitsforschung in der Region $\underline{\text { Augsburg. }}$

or early AMD at baseline $\left(\mathrm{eg},{ }^{23-26}\right.$ which is our late AMD incidence definition 2). It is useful particularly for association analyses, as it includes more individuals at baseline, more events during follow-up and options to model baseline early AMD as covariate. While the term 'incidence' might not be fully appropriate, because this type of event includes both, incidence and progression, it is used very often as such in published literature and a comparison should be facilitated. One reason beyond more events is maybe also a reduced issue by different early AMD classification systems: when no AMD and early AMD are taken together to define individuals at risk for late AMD, then different early AMD classification systems do not matter. We also derived late AMD incidence restricted to individuals AMD-free at baseline, our definition 1, which separates incidence from progression: the 3 -year risk estimates modelled from rates provide a practical guide for ophthalmology practitioners to help judge the late AMD risk for a person without early AMD (incident late AMD definition-1) versus a person with early AMD (progression). These estimates are subjected to uncertainty, as indicated by $95 \%$ CIs. Also, the modelling of yearly rates required an assumption on the time of onset, since we had only two AMD assessments per participant over time, but alternative onset times yielded similar results. As sample sizes particularly for late AMD were limited in the analyses per age-group, larger meta-analyses across cohort studies with harmonised early AMD classification, for example, within the European Eye Epidemiology consortium, are warranted to improve these estimates.

A challenge to compare estimates for incident early AMD and progression across published studies are the different classification systems for early AMD, with consequences also for the no AMD definition. We have previously highlighted substantial differences in early AMD prevalence for 3CACSS versus CC. ${ }^{7}$ Here, we document marked differences longitudinally: incidence for early AMD was lower, but progression to late AMD was 

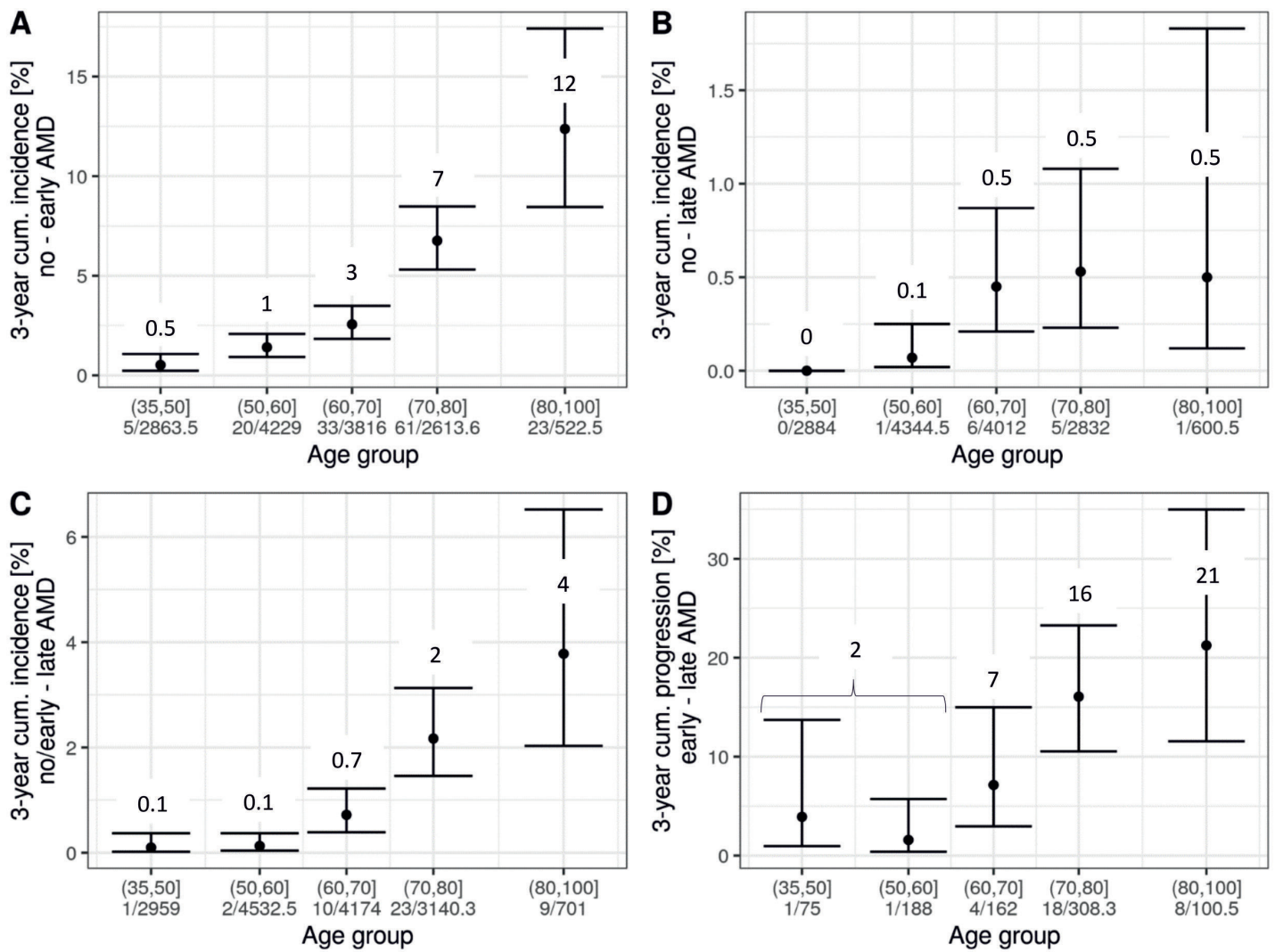

Figure 3 Three-year risk of incident early AMD, incident late AMD and progression by age groups in the joint analysis. In a joint analysis across the three studies (combining individuals from the same age groups as applicable), 3-year risk estimates by age groups were derived from rates assuming event onset in the middle of follow-up. Shown are estimates and $95 \% \mathrm{Cls}$ for (A) incident early AMD, (B) incident late AMD among individuals with no AMD at baseline (definition 1), (C) incident late AMD among individuals with no or early AMD at baseline (definition 2) and (D) progression from early to late AMD. Also stated are number of modelled events and person-years at risk. For AMD classification, 3CACSS was applied. Note the different y-axis scales. AMD, age-related macular degeneration; 3CACSS, Three Continent AMD Consortium Severity Scale.

higher for 3CACSS compared with CC, a pattern similarly as found by others. ${ }^{11}$ This and previous recommendation ${ }^{11}$ prompted us to focus on 3CACSS for most analyses. The different classification systems did not affect incident late AMD definition 2, which was expected, since the group at risk combines no with early AMD and 3CACSSbased early AMD is basically a subgroup of CC-based early $\mathrm{AMD}^{7}$; furthermore, they did not affect incident late AMD definition 1, despite the different groups at risk defined as absence of early or late AMD, probably because CC contributed little additional to 3CACSS in terms of prediction.

When comparing our results on late AMD incidence rate definition 2 with the recently published meta-analysis of four European cohorts in 7223 individuals, ${ }^{2}$ we found the $95 \%$ CIs to overlap: our rates per 1000 person-years were 2.2 (95\% CI 1.0 to 4.5 ) in 55-year old to 65 -year old and 11.7 (95\% CI 7.8 to 16.8 ) among 70-year to 100 -year old; the published meta-analysis yielded 0.5 (95\% CI 0.1 to 2.7) for individuals 50-70 years and 6.7 (95\% CI 3.2 to 14.1) for 70-100 years. For a more direct comparison, we gathered age-group-specific estimates from the meta-analysis' authors ${ }^{2}$ and embedded our three studies' rates. This yielded incidence rates from overall 8736 individuals covering 35 to 100 years, with the unique estimates in the 35-50-year old from our KORA-Fit study (figure 4). These estimates showed the expected increase by age with considerable variability between studies. To our knowledge, this provides the most comprehensive joint view on late AMD incidence from international population-based cohort studies so far.

Not included in the European meta-analysis was the Copenhagen City Eye Study ${ }^{27}(\mathrm{n}=359$, age 60-80 years, Wisconsin Age-Related Maculopathy Grading System): their 14-year cumulative incidence of early and late AMD (definition 2) of $31.5 \%$ and $14.8 \%,{ }^{27}$ respectively, were higher than our $13.9 \%$ and $4.6 \%$ from KORA FF4 14-year follow-up (slightly younger: 54-75 years, $n=350$, 3CACSS grading). Worldwide, the only two other long-term AMD studies, Beaver Dam Eye Study $^{26}$ and Blue Mountains Eye Study ${ }^{25}$ ( $n=3917$ and 1149 , age $43-86$ and $49+$ years, respectively), reported 15 -year cumulative incidence of $14.3 \%$ and $22.7 \%$ for early AMD and $3.1 \%$ and $6.8 \%$ for late AMD (definition 2), ${ }^{25}{ }^{26}$ which compared well to our KORA FF4 estimates. The Gutenberg Health Study, the only other population-based cohort from Germany ${ }^{9} \quad(n=6492$, age 35-74 years), yielded a 5-year cumulative any 


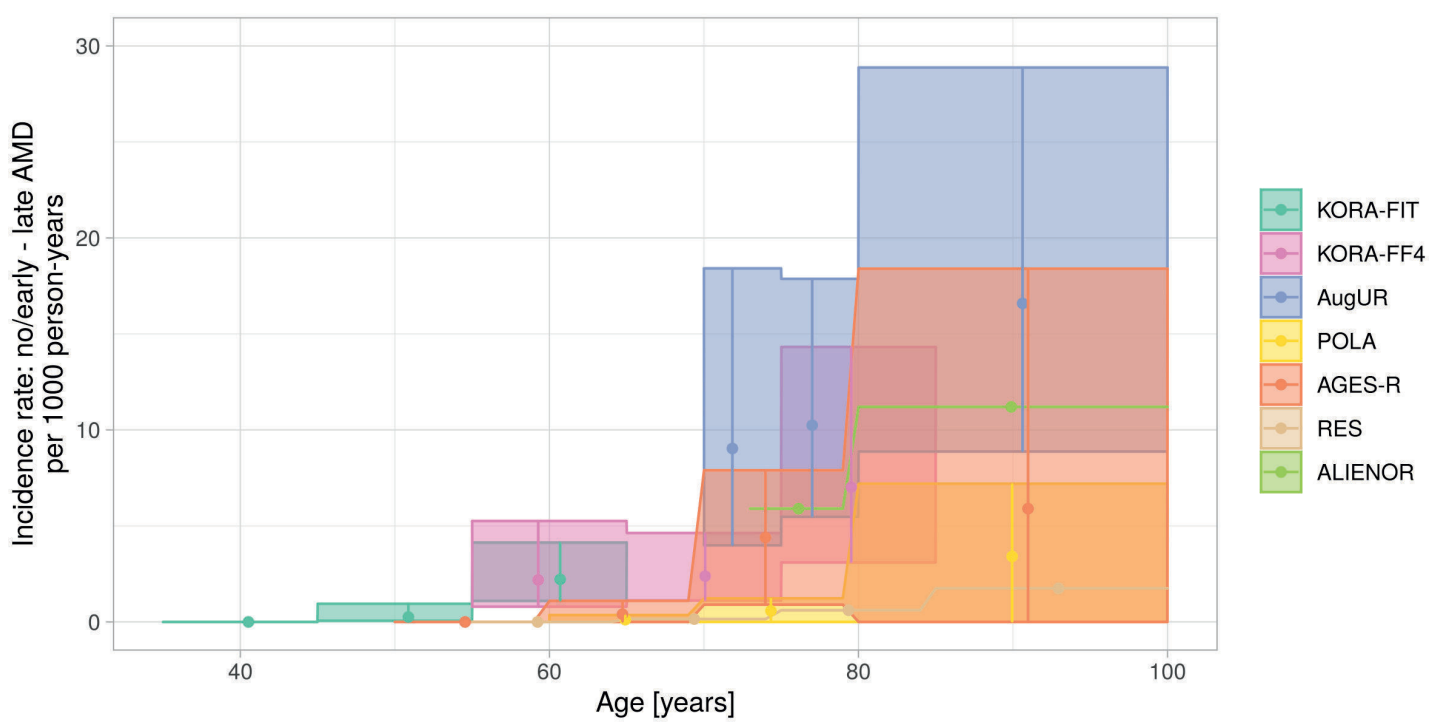

Figure 4 Comparison of European incidence rates for late AMD among individuals without or with early AMD at baseline. Depicted are estimated incidence rates per 1000 person-years and 95\% Cls for incident late AMD among individuals with no or early AMD at baseline. The four longitudinal studies included in the meta-analysis of Li et al ${ }^{2}$ are compared with KORA-Fit, KORA-FF4 and augur results (as in figure 2C). AGES-R, Age, Gene/Environment Susceptibility-Reykjavik Study; ALIENOR, Antioxydants, Lipids Essentiels, Nutrition et Maladies Oculaires Study; AMD, age-related macular degeneration;

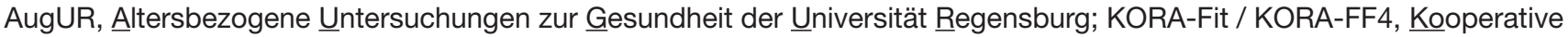
Gesundheitsforschung in der Region Augsburg; POLA, Pathologies Oculaires Liées à l'Age Study; RES, Rotterdam Eye Study.

AMD incidence among individuals AMD free at baseline $(1.7 \%, 69$ early or late AMD events); our longer follow-up in the younger/older and the inclusion of old aged enabled us to report on two times as many any AMD events $(n=142)$ despite a smaller sample size. Population-based cohort studies on progression cover diverse aspects of transition to more severe stages. ${ }^{24-26}$

Clinically very important is our observation that a relevant fraction, $22.7 \%$, of the 35-year to 55-year-old with 3CACSS-based early AMD at baseline progressed to late AMD within 18 years of follow-up. This suggests that the baseline-identified features in these relatively young individuals were not purely phenotypes mimicking AMD or other preceding retinal changes, but also reflected-at least in part- 'truly' early AMD, and that AMD is not solely relevant at old age. Our data demonstrate that these 35-55-year old with baseline early AMD exhibited at least a sevenfold increased 18-year cumulative risk for late AMD compared with individuals at the same age without AMD. As we had only 22 individuals with early AMD at that age, which was also reflected in the broad $95 \% \mathrm{CI}$, further studies are warranted. If substantiated, this should help draw attention to critical early AMD in the moderately aged and their risk for progression. This may be pivotal for timely diagnosis and therapy to help prevent vision loss in the middle of life.

Our association analyses were systematic for incident early AMD, incident late AMD definition-2, as well as progression. We confirmed known risk factors: age, genetic profile and early AMD at baseline. ${ }^{28} 29$ As the GRS was consistently significantly associated with increased odds for incident early and incident late AMD as well as for progression in old aged, our data support the potential usefulness of genetic testing in subjects with early AMD to help identify individuals at particularly high risk for progression to late AMD. We found smoking with a remarkably high cumulative 3-year risk for progression in individuals aged 70+, but only for current smoking at baseline ( $\mathrm{OR}=7.2,95 \%$ CI (1.0 to 67.2), $\mathrm{p}=0.06)$ and not for former smoking. This renders help to stop smoking a potentially effective prevention of progression also at old age. Limited number of events possibly hampered the detection of HDL-C association, ${ }^{28}$ but power is an issue for virtually all AMD cohort studies when analysed separately. We also found no association of sex and AMD incidence/progression. In the published literature, HDL-C and sex are both highly debated risk factors, mostly based on cross-sectional studies: some studies have implicated female sex or high HDL-C as associated with AMD, some have not and some have shown the opposite. ${ }^{30-33}$ Regarding longitudinal data, the Beaver Dam Eye $\mathrm{Study}^{34}$ or the Blue Mountains Eye Study ${ }^{25}$ showed female sex as a risk factor for early AMD incidence, but not for late AMD incidence; also, for example, not in the whole study population of the Beaver Dam Eye Study but only in women 75 years of age or older. Importantly, a recent approach using longitudinal data to develop a prediction model for incident late AMD applies a machine learning algorithm that allows the selection of the most predictive risk factors automatically. This approach also did not detect/include sex or HDL-C. ${ }^{29}$

Some limitations warrant mentioning: we need to acknowledge a selection at baseline towards the mobile elderly population in our old-aged AugUR participants due to the recruitment strategy; participants 
were required to come to the study centre, to conduct a 3-hour study programme, and to answer interview questions personally. ${ }^{7}$ However, follow-up response was high at $71 \%$ and baseline AMD frequencies did not differ between follow-up participants and drop outs. With regards to AMD assessment, the utilisation of colour fundus photography rather than optical coherence tomography or further multimodal imaging is a noteworthy limitation: smaller lesions might have been missed; multimodal imaging data can provide more and better reproducible information. Shorter follow-up intervals would have improved the uncertainty in estimating incidence rates. Missingness of image data for one of the two eyes of a participant can lead to biased estimates. ${ }^{735}$ However, the one-eye missingness was only $4 \%$ at baseline and $5 \%$ at follow-up and the bias, thus, limited.

In summary, we addressed incidence of early and late AMD as well as progression and provide results covering a particularly wide age spectrum. Our results in younger adults contribute uniquely to the existing literature and our joint view integrating our three studies' results to four other European cohorts is, to our knowledge, the most comprehensive view on incident late AMD to date. Our practical guide for 3-year risk of AMD incidence and progression is relevant for healthcare management, clinical trial design and patient counselling: given the age and existence/non-existence of early AMD, an ophthalmologist can judge the individual's risk to develop late AMD within 3 years. Large joint efforts to combine cohort study data are warranted to decrease uncertainty in estimates. While it is already routine practice to pay attention to individuals with early AMD $\geq 50$ years of age to enable timely diagnosis and therapy for late AMD, our work may help to extend this attention to early AMD in individuals aged $<50$ years.

\section{Author affiliations}

${ }^{1}$ Department of Genetic Epidemiology, University of Regensburg, Regensburg, Germany

2Department of Ophthalmology, University Hospital Regensburg, Regensburg, Germany

${ }^{3}$ Statistical Consulting Unit StaBLab, Department of Statistics, Ludwig Maximilians University Munich, Munich, Germany

${ }^{4}$ Department of Ophthalmology, University Hospital Augsburg, Augsburg, Germany

${ }^{5}$ Institute for Epidemiology, Helmholtz Zentrum München Deutsches

Forschungszentrum für Gesundheit und Umwelt, Neuherberg, Germany

${ }^{6}$ KORA Study Centre, University Hospital Augsburg, Augsburg, Germany

${ }^{7}$ Chair of Epidemiology, Institute for Medical Information Processing, Biometry and

Epidemiology, Medical Faculty, Ludwig Maximilians University Munich, Munich,

Germany

${ }^{8}$ Department of Ophthalmology, University Hospital Bonn, Bonn, Germany

${ }^{9}$ Institute of Human Genetics, University of Regensburg, Regensburg, Germany

Acknowledgements We gratefully acknowledge the excellent supporting assistance of Lydia Mayerhofer, Magdalena Scharl, Josef Simon and Sylvia Pfreintner. Moreover, we thank all study participants for contributing to the KORA and AugUR study.

Contributors CB: eye sub-study coordinator for AugUR and KORA, manuscript writing, grading of colour fundus images, statistical analysis, interpreting results. FG: advanced statistical modelling, manuscript writing. MEZ: data management, statistical analysis. KIH: imaging and processing of KORA-Fit participants with non-gradable images in study centre. GE: imaging and processing of KORA-Fit participants with non-gradable images in study center. TB: second grading of colour fundus images. BL: KORA study conduct, data management. MH: KORA study conduct. AP: KORA study principal investigator, scientific oversight over KORA study conduct. JQL: providing refined age group specific incidence estimates from meta-analysis. RPF: providing refined age group specific incidence estimates from meta-analysis, manuscript writing. $\mathrm{HH}$ : manuscript writing, ophthalmological expertise. BHFW: AugUR study initiation and supervision, manuscript writing. HK: manuscript writing, advanced statistical modelling. AM: manuscript writing, ophthalmological expertise. KJS: AugUR study coordinator, manuscript writing, data management, statistical analysis, interpreting results. IMH: AugUR study principal investigator, project initiation and supervision, manuscript writing, interpreting results. Guarantor: IMH. All authors contributed to the reviewing and editing of the manuscript.

Funding The KORA study was initiated and financed by the Helmholtz Zentrum München-German Research Centre for Environmental Health, which is funded by the German Federal Ministry of Education and Research (BMBF) and by the State of Bavaria. The AugUR study and analyses are supported by grants from the German Federal Ministry of Education and Research (BMBF 01ER1206, BMBF 01 ER1507 to IMH), by the Deutsche Forschungsgemeinschaft (DFG, German Research Foundation; HE 3690/7-1 and HE 3690/5-1 to IMH, BR 6028/2-1 to CB), by the National Institutes of Health (NIH R01 EY RES 511967 to IMH.), by the PRO RETINA Foundation (Brandl.1-2017 to CB) and institutional budget (Institute of Human Genetics, Department of Genetic Epidemiology, University of Regensburg).

Disclaimer The sponsors or funding organisations had no role in the design or conduct of this research.

Competing interests TB is a member of the advisory board of Novartis; this is unrelated to the work presented here. RPF is a consultant for Novartis, Bayer, Roche/Genentech, Allergan, Alimera, Böhringer-Ingelheim, Chiesi, ProQR, Opthea and Inositec, and receives research support from Novartis, Biogen, Zeiss Meditec Heidelberg Engineering and Centrevue; none of this has any relation to the presented work. HH received honoraria for lectures and advisory boards unrelated to this work from Alcon, Allergan, Apellis, Bayer, Novartis and Theapharm. BHFW has cooperation agreements for projects unrelated to this work with Alcon, Allergan, Apellis, Gensight Biologics, Novartis, Shire, F. Hoffmann-La Roche and ModernaTX, and is a member of the clinical advisory board of Gyroscope Therapeutics. IMH has received support from Roche Diagnostics for a project related to age-related macular degeneration, but unrelated to this work presented here. No conflicting relationship exists for the other authors.

Patient consent for publication Consent obtained directly from patient(s)

Ethics approval This study involves human participants and was approved by The Ethics Committee of the Bavarian Chamber of Physicians, Munich, approved the KORA study including its baseline and follow-up surveys (KORA-S4: EC Number 99186, for genetic epidemiological questions 05004; KORA FF4: EC Number 06068; KORA-Fit: EC Number 17040), which also comply with the Declaration of Helsinki and its later amendments. All participants gave written informed consent for participation in the study. The AugUR study was approved by the Ethics Committee of the University of Regensburg, Germany (vote 12-101-0258). The study complies with the Declaration of Helsinki and its later amendments. All participants provided informed written consent. Participants gave informed consent to participate in the study before taking part.

Provenance and peer review Not commissioned; externally peer reviewed.

Data availability statement Data are available upon reasonable request. The datasets generated and analysed are not publicly available due to data privacy of study participants, but are available upon reasonable request.

Supplemental material This content has been supplied by the author(s). It has not been vetted by BMJ Publishing Group Limited (BMJ) and may not have been peer-reviewed. Any opinions or recommendations discussed are solely those of the author(s) and are not endorsed by BMJ. BMJ disclaims all liability and responsibility arising from any reliance placed on the content. Where the content includes any translated material, BMJ does not warrant the accuracy and reliability of the translations (including but not limited to local regulations, clinical guidelines, terminology, drug names and drug dosages), and is not responsible for any error and/or omissions arising from translation and adaptation or otherwise.

Open access This is an open access article distributed in accordance with the Creative Commons Attribution Non Commercial (CC BY-NC 4.0) license, which permits others to distribute, remix, adapt, build upon this work non-commercially, and license their derivative works on different terms, provided the original work is 
properly cited, appropriate credit is given, any changes made indicated, and the use is non-commercial. See: http://creativecommons.org/licenses/by-nc/4.0/.

\section{ORCID iDs}

Caroline Brandl http://orcid.org/0000-0001-8223-6137

Martina E Zimmermann http://orcid.org/0000-0002-6916-4404

Jeany Q Li http://orcid.org/0000-0002-9771-3431

\section{REFERENCES}

1 Lim LS, Mitchell P, Seddon JM, et al. Age-related macular degeneration. Lancet 2012;379:1728-38.

2 Li JQ, Welchowski T, Schmid M, et al. Prevalence and incidence of age-related macular degeneration in Europe: a systematic review and meta-analysis. Br J Ophthalmol 2020;104:1077-84.

3 Wong WL, Su X, Li X, et al. Global prevalence of age-related macular degeneration and disease burden projection for 2020 and 2040: a systematic review and meta-analysis. Lancet Glob Health 2014;2:e106-16.

4 Colijn JM, Buitendijk GHS, Prokofyeva E, et al. Prevalence of agerelated macular degeneration in Europe: the past and the future. Ophthalmology 2017;124:1753-1763.

5 Korb CA, Kottler UB, Wolfram C, et al. Prevalence of age-related macular degeneration in a large European cohort: results from the population-based Gutenberg health study. Graefes Arch Clin Exp Ophthalmol 2014;252:1403-11.

6 Brandl C, Breinlich V, Stark KJ, et al. Features of age-related macular degeneration in the general adults and their dependency on age, sex, and smoking: results from the German KorA study. PLoS One 2016;11:e0167181.

7 Brandl C, Zimmermann ME, Günther F, et al. On the impact of different approaches to classify age-related macular degeneration: results from the German augur study. Sci Rep 2018;8:1-10.

8 Tomany SC, Wang JJ, Van Leeuwen R, et al. Risk factors for incident age-related macular degeneration: pooled findings from 3 continents. Ophthalmology 2004;111:1280-7.

9 Fieß A, Elbaz H, Korb CA, et al. Low birth weight is linked to age-related macular degeneration: results from the populationbased Gutenberg health study (GHS). Investig Ophthalmol Vis Sci 2019;60:4943-50.

10 Spaide RF, Jaffe GJ, Sarraf D, et al. Consensus Nomenclature for reporting neovascular age-related macular degeneration data: consensus on neovascular age-related macular degeneration nomenclature Study Group. Ophthalmology 2020;127:616-36.

11 Thee EF, Meester-Smoor MA, Luttikhuizen DT, et al. Performance of classification systems for age-related macular degeneration in the Rotterdam study. Trans/ Vis Sci Technol 2020;9:26.

12 Klein R, Meuer SM, Myers CE, et al. Harmonizing the classification of age-related macular degeneration in the three-continent AMD Consortium. Ophthalmic Epidemiol 2014;21:14-23.

13 Ferris FL, Wilkinson CP, Bird A, et al. Clinical classification of agerelated macular degeneration. Ophthalmology 2013;120:844-51.

14 Stark K, Olden M, Brandl C, et al. The German augur study: study protocol of a prospective study to investigate chronic diseases in the elderly. BMC Geriatr 2015;15:130.

15 Fritsche LG, Igl W, Bailey JNC, et al. A large genome-wide association study of age-related macular degeneration highlights contributions of rare and common variants. Nat Genet 2016;48:134-43.
16 Winkler TW, Grassmann F, Brandl C, et al. Genome-Wide association meta-analysis for early age-related macular degeneration highlights novel loci and insights for advanced disease. BMC Med Genomics 2020;13:120-18.

17 Holle R, Happich M, Löwel H, et al. KORA - A Research Platform for Population Based Health Research. Gesundheitswesen 2005;67:19-25.

18 Wichmann H-E, Gieger C, Illig T, et al. KORA-gen--resource for population genetics, controls and a broad spectrum of disease phenotypes. Gesundheitswesen 2005;67 Suppl 1:26-30.

19 Stratmann B, Xu T, Meisinger C, et al. PLA1A2 platelet polymorphism predicts mortality in prediabetic subjects of the population based KorA S4-Cohort. Cardiovasc Diabetol 2014;13:90.

20 Kowall B, Rathmann W, Stang A, et al. Perceived risk of diabetes seriously underestimates actual diabetes risk: the KorA FF4 study. PLoS One 2017;12:e0171152-75.

21 Peters A, Döring A, Ladwig K-H. Multimorbidität und erfolgreiches Altern. Z Gerontol Geriatr 2011;44.

22 Holle R, Happich M, Löwel H, et al. KORA--a research platform for population based health research. Gesundheitswesen 2005;67 Suppl 1:19-25.

23 Delcourt C, Lacroux A. Related Macular Degeneration : The “ Pathologies Oculaires Liées I " Age ". Specialist 2005:924-6.

24 Klaver CC, Assink JJ, van Leeuwen R, et al. Incidence and progression rates of age-related maculopathy: the Rotterdam study. Invest Ophthalmol Vis Sci 2001;42:2237-41.

25 Joachim N, Mitchell P, Burlutsky G, et al. The incidence and progression of age-related macular degeneration over 15 years: the blue Mountains eye study. Ophthalmology 2015;122:2482-9.

26 Klein R, Klein BEK, Knudtson MD, et al. Fifteen-year cumulative incidence of age-related macular degeneration: the Beaver dam eye study. Ophthalmology 2007;114:253-62.

27 Buch H, Nielsen NV, Vinding T, et al. 14-Year incidence, progression, and visual morbidity of age-related maculopathy: the Copenhagen City eye study. Ophthalmology 2005;112:787-98.

28 Heesterbeek TJ, Lorés-Motta L, Hoyng CB, et al. Risk factors for progression of age-related macular degeneration. Ophthalmic Physiol Opt 2020;40:140-70.

29 Ajana S, Cougnard-Grégoire A, Colijn JM, et al. Predicting Progression to Advanced Age-Related Macular Degeneration from Clinical, Genetic, and Lifestyle Factors Using Machine Learning. Ophthalmology 2021;128:587-97.

30 Mitchell P, Smith W, Attebo K, et al. Prevalence of age-related maculopathy in Australia. the blue Mountains eye study. Ophthalmology 1995;102:1450-60

31 Friedman DS, O'Colmain BJ, Muñoz B, et al. Prevalence of agerelated macular degeneration in the United States. Arch Ophthalmol 2004;122:564-72.

32 Fraser-Bell S, Donofrio J, Wu J, et al. Sociodemographic factors and age-related macular degeneration in Latinos: the Los Angeles Latino eye study. Am J Ophthalmol 2005;139:30-8.

33 Colijn JM, den Hollander Al, Demirkan A, et al. Increased highdensity lipoprotein levels associated with age-related macular degeneration: evidence from the EYE-RISK and European eye epidemiology consortia. Ophthalmology 2019;126:393-406.

34 Klein R, Klein BE, Jensen SC, et al. The five-year incidence and progression of age-related maculopathy: the Beaver dam eye study. Ophthalmology 1997;104:7-21.

35 Günther F, Brandl C, Heid IM, et al. Response misclassification in studies on bilateral diseases. Biom J 2019;61:1033-1048. 